\title{
BMJ Haemodynamic and structural correlates open of the first and second heart sounds in pulmonary arterial hypertension: an acoustic cardiography cohort study
}

\author{
William Chan,, ${ }^{1,2}$ Maryam Woldeyohannes, ${ }^{1,2}$ Rebecca Colman, ${ }^{2,5}$ Patti Arand, ${ }^{3}$ \\ Andrew D Michaels, ${ }^{4}$ John D Parker, ${ }^{1,2}$ John T Granton, ${ }^{2,5}$ Susanna Mak ${ }^{1,2}$
}

To cite: Chan W,

Woldeyohannes M,

Colman R, et al.

Haemodynamic and structural correlates of the first and second heart sounds in pulmonary arterial

hypertension: an acoustic cardiography cohort study. BMJ Open 2013;3:e002660. doi:10.1136/bmjopen-2013002660

- Prepublication history for this paper are available online. To view these files please visit the journal online (http://dx.doi.org/10.1136/ bmjopen-2013-002660).

Received 30 January 2013 Revised 10 March 2013 Accepted 11 March 2013

This final article is available for use under the terms of the Creative Commons Attribution Non-Commercial 2.0 Licence; see http://bmjopen.bmj.com

For numbered affiliations see end of article.

Correspondence to Dr Susanna Mak; smak@mtsinai.on.ca

\section{ABSTRACT}

Objective: To examine the relationship between acoustic characteristics of the first and second heart sounds (S1 and S2) and underlying cardiac structure and haemodynamics in patients with isolated pulmonary arterial hypertension (PAH) and controls.

Design: Prospective multicentre cohort study. Setting: Tertiary referral and community hospitals. Participants: We prospectively evaluated $40 \mathrm{PAH}$ patients undergoing right-heart catheterisation with contemporaneous digital acoustic cardiography (intensity and complexity) and two-dimensional transthoracic echocardiography. To normalise for differences in body habitus, acoustic variables were also expressed as a ratio (S2/S1). 130 participants (55 also had haemodynamic and/or echocardiographic assessment) without clinical or haemodynamic evidence of PAH or congestive heart failure acted as controls.

Results: Patients with PAH had higher mean pulmonary artery pressure (mPA; $40 \pm 13$ vs $16 \pm 4 \mathrm{~mm} \mathrm{Hg}, p<0.0001)$ and pulmonary vascular resistance $(9 \pm 6$ vs $1 \pm 1$ Wood Units, $p<0.0001)$ compared with controls, but cardiac index and mean pulmonary capillary wedge pressure were similar. More PAH patients had evidence of right ventricular (RV) dilation $(50 \%$ vs $19 \%)$ and RV systolic dysfunction $(41 \%$ vs $9 \%)$ in the moderate-severe range (all $p<0.05)$. Compared with controls, the acoustic profiles of PAH patients were characterised by increased $\mathrm{S} 2$ complexity, S2/S1 complexity and S2/S1 intensity (all $p<0.05)$. In the PAH cohort, $S 2$ complexity was inversely related to $\mathrm{S} 1$ complexity. MPA was the only independent multivariate predictor of S2 complexity. The severity of RV enlargement and systolic impairment had reciprocal effects on the complexity of S2 (increased) and S1 (decreased). Decreased S1 complexity was also related to evidence of a small left ventricular cavity.

Conclusions: Acoustic characteristics of both $\mathrm{S} 1$ and S2 are related to the severity of PAH and are associated with RV enlargement and systolic dysfunction. The reciprocal relationship between S2 and $\mathrm{S} 1$ complexity may also reflect the underlying ventricular interaction associated with PAH.

\section{ARTICLE SUMMARY}

Article focus

- To examine the relationship between acoustic characteristics of the first and second heart sounds (S1 and S2) and underlying cardiac structure and haemodynamics in patients with isolated pulmonary arterial hypertension (PAH) and controls.

- The changes in cardiac structure and haemodynamics with PAH can be detected by quantitative acoustic cardiography.

Key messages

- The normal acoustic profile observed in controls is altered in patients with PAH.

- Acoustic properties of S2 were directly related to invasively derived mean pulmonary artery pressure and pulmonary vascular resistance.

- The reciprocal relationship between S1 and S2 among patients with PAH may reflect the phenomenon of ventricular interaction that develops as the right ventricle remodels during the course of PAH.

Strengths and limitations of this study

- Prospective multicentre study with detailed acoustic recording and correlation with echocardiographic and haemodynamic parameters.

- Relatively small sample size of the control and PAH cohorts.

\section{INTRODUCTION}

Pulmonary arterial hypertension (PAH) is characterised by increased pulmonary vascular resistance (PVR) and consequent right ventricular (RV) failure due to pressure overload. ${ }^{12}$ Once considered a rare disease, $\mathrm{PAH}$ is recognised as a complication of a spectrum of clinical conditions. ${ }^{3}$ Recent advances in treatment have intensified the interest in identifying afflicted patients. ${ }^{4}$

The physical examination is the clinician's first opportunity for the objective assessment 
of a patient's haemodynamic state. Traditional pedagogy states that abnormal intensity, quality and splitting of the second heart sound (S2) are auscultatory signs of pulmonary hypertension (PH). ${ }^{5}$ However, physical signs of $\mathrm{PH}$ were often evaluated in the context of confounding cardiac abnormalities such as left-to-right shunting or left ventricular (LV) systolic dysfunction. Moreover, quantitative relationships between auscultatory findings and derangement of the RV-pulmonary arterial circulatory unit have not been well characterised. ${ }^{6}$ Digital acoustic cardiography permits quantitative analysis of precordial heart sounds. ${ }^{7}$ Our aim was to quantitatively examine the precordial acoustic profile as it relates to cardiac haemodynamic and structural alterations in patients with isolated PAH and controls.

\section{METHODS}

\section{Study participants}

This was a prospective, multicentre, observational cohort study. The PAH cohort was recruited from the PH clinic of the University Health Network. All patients routinely undergo transthoracic two-dimensional echocardiogram (2D-TTE) and Doppler examination, measurements of B-type natriuretic peptide and 6 min walk tests. Patients with suspected or established PAH are referred for haemodynamic assessment. Consecutive patients referred for right-heart catheterisation (RHC) between November 2010 and July 2011 were invited to participate. Exclusion criteria included the echocardiographic determination of LV systolic dysfunction or valvular heart disease (suspected WHO group II $\mathrm{PH}$ ) given possible acoustic abnormalities related to left heart disease. Patients with chronic lung diseases (suspected WHO group III PH) were also excluded, as abnormalities of pulmonary function may confound the transmission of acoustic signals. Patients were also excluded if a non-sinus rhythm was identified or if complete right or left bundle branch block was observed (QRS duration $>120 \mathrm{~m}$ ).

The control group consisted of individuals in sinus rhythm without clinical or haemodynamic evidence of $\mathrm{PH}$ or congestive heart failure. Healthy volunteers without a history of cardiac or pulmonary diseases were recruited from the community and underwent acoustic cardiography alone $(n=75)$. A second group of controls without $\mathrm{PH}$ were derived from patients who underwent acoustic cardiography and 2D-TTE and/or RHC $(n=55)$ for the evaluation of chest pain syndromes.

The study protocols for the PAH cohort were approved by the Mount Sinai Hospital Research Ethics Board (approval number 10-0142-E) and by local US institutional review boards for the control cohorts (University of Utah, approval number was 26801; University of California, San Francisco approval number H8831-22924; and Western Institutional Review Board, Washington, approval number 1037785). All participants provided written informed consent.
Cardiac catheterisation procedures and haemodynamic data

RHC was performed with standard balloon floatation pulmonary artery catheter in the fasting state, without sedation. Haemodynamic measurements were recorded at end-expiration and included heart rate, systolic/diastolic/mean arterial pressure (SAP/DAP/MAP), right atrial pressure, pulmonary artery systolic/diastolic/mean pressures (PASP/PADP/mPA) and pulmonary capillary wedge pressure (PCWP). Cardiac output was measured using both the Fick method and in triplicate by thermodilution. PVR and systemic vascular resistances were calculated using standard formulas.

\section{Transthoracic echocardiography}

The 2D-TTE assessment was performed within a week of the RHC. LV ejection fraction, end-diastolic and endsystolic volumes were calculated using the biplane method of disc summation and indexed to BSA. ${ }^{8}$ Semiquantitative and qualitative assessment of RV structure and function was performed following review of standard 2D acoustic windows that included RV inflow and outflow tracts, parasternal short-axis views and apical views. Parameters of RV size (at end-diastole from an RV-focused apical fourchamber view) and RV systolic function (at least one of the fractional area change and tricuspid annular plane systolic excursion) were used in the semiquantitative analysis of RV structure and function.

\section{Digital acoustic cardiography and ECG analysis}

Acoustic cardiography was performed on the same day as the RHC with the Audicor device (Inovise Medical, Inc, Beaverton, Oregon, USA), either prior to or following RHC with the patient supine with head incline of $30^{\circ}$ during tidal respiration. Standard 12-lead placement was performed with two dual-function (audioelectrocardiographic) sensors in the precordial V3 and V4 positions capable of simultaneously capturing cardiac electrical and acoustic signals. Five $10 \mathrm{~s}$ artefact-free recordings were obtained and analysed utilising proprietary software. This algorithm utilises wavelet-based signal processing techniques for acoustic analysis. Time-frequency analysis is performed, permitting separation of heart sounds from murmurs and artefacts. The Audicor algorithm reduces the confounders caused by external noise, speech, movement and permits analysis in individuals with high body mass index. ${ }^{7}$

Two acoustic cardiographic characteristics of S1 and S2 were analysed: intensity and complexity. A value for heart sound intensity is generated based on the peak-to-peak amplitude of the sound and expressed in $\mathrm{mV}$ units. Heart sound complexity is a correlate of the auditory perception of valve sound crispness and is determined using time-frequency measures of width, intensity and frequency content of the signal, which is influenced by valve splitting or closure abnormalities. Based on spectral analysis, complexity is expressed as a dimensionless index. Figure 1 demonstrates the 
Figure 1 Acoustic cardiographic output for a control patient $(A)$ and a patient with pulmonary arterial hypertension (PAH) $(\mathrm{B})$ showing real-time sound trace, scalogram time-frequency representation, filter bands and real-time ECG trace. The scalogram time-frequency representation shows frequency on the vertical axis on a logarithmic scale from 5 to $220 \mathrm{~Hz}$ and time on the horizontal axis. The squared magnitude of the wavelet transform energy at each frequency is represented by colours ranging from dark blue (bottom $2 \%$ ) to deep red (top $2 \%$ ).

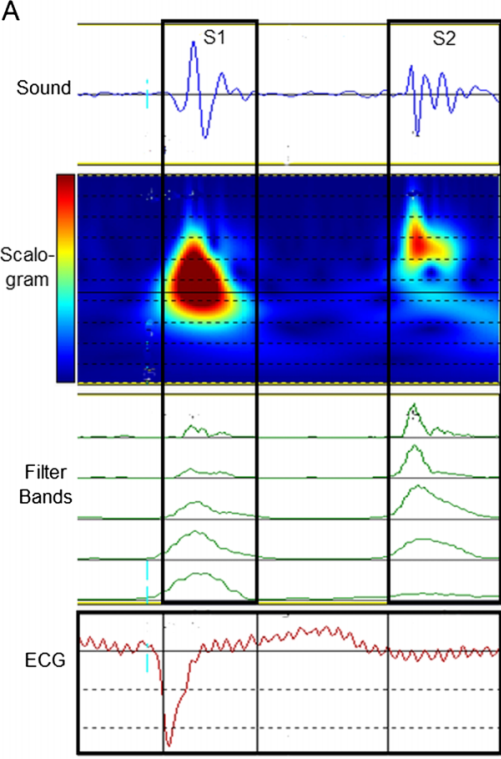

Control: V4 S1 complexity $3.7, \mathrm{~S} 2$ complexity 2.9

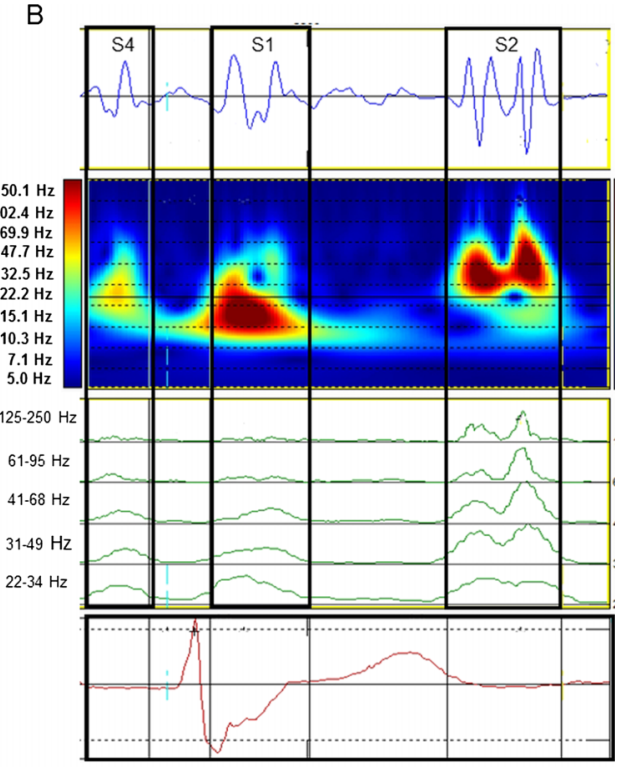

PAH: V4 S1 complexity 2.4, S2 complexity 9.3 representative acoustic recordings and spectral displays from a control and a PAH participant.

Heart sound intensity, and possibly the complexity, is known to be influenced by factors such as BSA, characteristics of the thoracic cage and cardiac axis. ${ }^{5}$ Therefore, complexity and intensity are expressed in absolute values as well as normalised and expressed as a ratio between $\mathrm{S} 2$ and $\mathrm{S} 1$ to account for variations in anthropomorphic characteristics.

\section{Statistical analysis}

Categorical data are presented as numbers and percentages. Continuous data are presented as mean \pm SD. Continuous variables were compared with either paired or unpaired Student t test, while categorical variables were compared between groups with Pearson $\chi^{2}$ test or a Fisher exact test where appropriate.

We performed univariate and multivariate linear logistic regression to examine the relationship between haemodynamic variables with $\mathrm{S} 1$ and $\mathrm{S} 2$ complexity. Binary logistic regression, for example, with $\mathrm{mPA} \geq 25 \mathrm{~mm} \mathrm{Hg}$ as the dependent variable, was performed to examine its association with audioelectrocardiographic variables. Receiver-operating characteristic (ROC) curves were then plotted using the univariate score for each patient allowing the calculation of sensitivity, specificity and the areaunder-the-ROC curve (AUC ROC).

All reported $p$ values are two-sided. The $p<0.05$ was considered statistically significant. Data analyses were performed with SPSS V.16 (SPSS Inc, Chicago, Illinois, USA).

\section{RESULTS}

\section{Study participants}

Forty patients were enrolled in the PAH cohort. The baseline characteristics of these patients are presented in table 1 . The predominant aetiology of PAH was classified as idiopathic, followed by that associated with underlying connective tissue disease. A total of 130 controls with acoustic cardiographic data were enrolled. Of these, 55 had contemporaneous echocardiographic data and 28 had RHC and echocardiographic data. Both groups were similar in age (PAH $58 \pm 16$ years, controls 59 $\pm 11, \mathrm{p}=0.73)$. A higher proportion of the $\mathrm{PAH}$ cohort were women $(78 \%$ vs $55 \%, \mathrm{p}<0.01)$ and thus the PAH cohort also exhibited smaller BSA $(1.7 \pm 0.2$ vs $\left.1.9 \pm 0.3 \mathrm{~m}^{2}, \mathrm{p}<0.01\right)$.

\section{Acoustic cardiography measurements}

Heart rate and $\mathrm{PR}$ intervals were similar between patients with $\mathrm{PAH}$ and controls. Although patients with complete right-bundle or left-bundle branch blocks were excluded, patients with $\mathrm{PAH}$ exhibited a slightly greater

Table 1 Baseline clinical characteristics of $\mathrm{PAH}$ patients

\begin{tabular}{ll}
\hline Classification of PAH (n) & PAH (40) \\
\hline Group 1 & \\
$\quad$ Idiopathic & 13 \\
Connective tissue disease & 21 \\
Portal hypertension & 2 \\
Other & 3 \\
Group 4 (chronic thromboembolic) & 1 \\
NYHA class & $2.4 \pm 0.6$ \\
6 min walk test (total distance) (m) & $423 \pm 96$ \\
B-type natriuretic peptide (pmol/l) & $345 \pm 612$ \\
Medications (\%) & \\
$\quad$ Endothelin receptor antagonists & 41 \\
Calcium channel antagonists & 28 \\
Phosphodiesterase V inhibitor & 40 \\
\hline NYHA, New York Heart Association; PAH, pulmonary arterial \\
hypertension.
\end{tabular}


Table 2 Acoustic cardiography measurements

\begin{tabular}{lccc}
\hline (n) & PAH (40) & Controls (130) & p Value \\
\hline PR interval (ms) & $156 \pm 45$ & $157 \pm 29$ & 0.94 \\
QRS duration (ms) & $105 \pm 21$ & $95 \pm 17$ & 0.04 \\
Heart rate (bpm) & $72 \pm 14$ & $70 \pm 11$ & 0.31 \\
S1 intensity & & & \\
V3 & $1.8 \pm 1.2^{*}$ & $2.3 \pm 1.9^{*}$ & 0.15 \\
V4 & $2.4 \pm 2.0 \dagger$ & $2.7 \pm 2.6 \dagger$ & 0.50 \\
S1 complexity & & & \\
V3 & $4.5 \pm 1.6 \ddagger$ & $4.5 \pm 2.5 \ddagger$ & 0.89 \\
V4 & $4.6 \pm 2.0 \S$ & $5.1 \pm 3.0 \S$ & 0.32 \\
S2 intensity & & & \\
V3 & $1.2 \pm 0.9^{*}$ & $1.2 \pm 1.1^{*}$ & 0.998 \\
V4 & $1.7 \pm 1.7 \dagger$ & $1.4 \pm 1.3 \dagger$ & 0.16 \\
S2 complexity & & & \\
V3 & $3.7 \pm 2.0 \ddagger$ & $1.5 \pm 1.1 \ddagger$ & $<0.0001$ \\
V4 & $4.6 \pm 4.7 \S$ & $1.3 \pm 1.1 \S$ & $<0.0001$ \\
S2/S1 intensity & & & \\
V3 & $0.73 \pm 0.28$ & $0.54 \pm 0.35$ & 0.02 \\
V4 & $0.74 \pm 0.28$ & $0.54 \pm 0.30$ & 0.01 \\
S2/S1 complexity & & & \\
V3 & $1.0 \pm 0.7$ & $0.5 \pm 0.5$ & $<0.0001$ \\
V4 & $1.3 \pm 1.7$ & $0.3 \pm 0.3$ & $<0.0001$ \\
\hline The
\end{tabular}

The italicized $p$ value refers to the between group comparisons (between columns or horizontally). The annotated values and legend refer to within group comparisons (within columns or vertically).

*PAH V3 S1 vs S2 intensity, $p=0.01$; control V3 S1 vs S2 intensity, $\mathrm{p}<0.0001$.

†PAH V4 S1 vs S2 intensity, $p=0.09$; control V4 S1 vs S2 intensity, $\mathrm{p}<0.0001$.

$\ddagger P A H ~ V 3 ~ S 1$ vs $S 2$ complexity, $p=0.07$; control V3 S1 vs $S 2$ complexity, $\mathrm{p}<0.0001$.

$\S \mathrm{PAH}$ V4 S1 vs S2 complexity, $p=0.998$; control V4 S1 vs S2 complexity, $p<0.0001$.

$\mathrm{PAH}$, pulmonary arterial hypertension

QRS duration. Acoustic characteristics of S1 and S2 in the PAH and control groups are presented in table 2.

Among the control group, both the intensity and complexity of S1 were increased compared with S2. In contrast, among the PAH group, the complexity of S2 was increased compared with S1. In the PAH group, the complexity of S2 in both precordial lead positions was also significantly increased compared with the control group. Thus, the S2/S1 complexity ratio was also highly significantly different between the PAH and control groups. Although S2 intensity was not different between $\mathrm{PAH}$ and control groups, the S2/S1 intensity ratio was significantly increased among patients with PAH compared with controls.

Haemodynamic measurements: relationship between acoustic cardiographic variables and pulmonary vascular haemodynamics

Haemodynamic and acoustic data were available from the $40 \mathrm{PAH}$ cohort and 28 control participants and are shown in table 3. As expected, patients with PAH demonstrated significantly increased $\mathrm{mPA}$ and PVR. Importantly, there were no significant differences in PCWP or cardiac index.
Table 3 Haemodynamic measurements

\begin{tabular}{lccc}
\hline & $\begin{array}{l}\text { PAH } \\
\text { (n) }\end{array}$ & $\begin{array}{l}\text { Controls } \\
\mathbf{( 2 8 )}\end{array}$ & p Value \\
\hline $\begin{array}{l}\text { Mean right atrial pressure } \\
\text { (mm Hg) }\end{array}$ & $5 \pm 4$ & $3 \pm 4$ & 0.13 \\
$\begin{array}{l}\text { Pulmonary artery systolic } \\
\text { pressure (mm Hg) }\end{array}$ & $68 \pm 23$ & $25 \pm 6$ & $<0.0001$ \\
$\begin{array}{l}\text { Pulmonary artery diastolic } \\
\text { pressure (mm Hg) }\end{array}$ & $22 \pm 8$ & $11 \pm 16$ & 0.001 \\
$\begin{array}{l}\text { Mean pulmonary artery } \\
\text { pressure (mm Hg) }\end{array}$ & $40 \pm 13$ & $16 \pm 4$ & $<0.0001$ \\
$\begin{array}{l}\text { Mean pulmonary capillary } \\
\text { wedge pressure (mm Hg) }\end{array}$ & $8 \pm 4$ & $9 \pm 3$ & 0.37 \\
$\begin{array}{l}\text { Cardiac index (Fick) } \\
\text { (I/min/m }{ }^{2} \text { ) }\end{array}$ & $2.4 \pm 0.7$ & $2.5 \pm 1.0$ & 0.54 \\
$\begin{array}{l}\text { Pulmonary vascular } \\
\text { resistance (wood unit) }\end{array}$ & $9 \pm 6$ & $1 \pm 1$ & $<0.0001$ \\
\hline $\begin{array}{l}\text { PAH, pulmonary arterial hypertension. } \\
\text { (2) }\end{array}$ & & & \\
\end{tabular}

As the complexity of S2 was significantly increased in the PAH group, we examined the relationship between S2 complexity and pulmonary vascular haemodynamics in the entire cohort. As shown in figure 2, both V3 and V4 S2 complexity correlated significantly with $\mathrm{mPA}$ and PVR. We examined the relationship between S2 complexity and other haemodynamic variables including heart rate, cardiac output, right atrial pressure, PCWP, mean systemic arterial pressure and $\mathrm{mPA}$ (table 4). Mean PA pressure was the only independent multivariate predictor for S2 complexity in both precordial lead positions.

In contrast to $\mathrm{S} 2$ complexity, we observed that $\mathrm{mPA}$ was inversely correlated with V4 S1 complexity. Cardiac output was a univariate predictor of S1 complexity in the both the V3 and V4 positions. The relationship between cardiac output and V3 S1 complexity remained significant after multivariable analysis of other haemodynamic variables.

\section{Echocardiographic measurements: relationship between} acoustic cardiographic variables and RV enlargement and systolic dysfunction

Echocardiographic data were available for all the PAH group and the 28 controls who also had haemodynamic data. As expected, compared with the control group, the RVSP was significantly elevated and a larger portion of the PAH group had evidence of RV dilation (50\% vs $19 \%, \mathrm{p}=0.02$ ) and RV systolic dysfunction ( $41 \%$ vs $9 \%, \mathrm{p}=0.01)$ in the moderate-to-severe range on echocardiography.

We examined the relationship between S2 and S1 complexity based on the abnormalities of RV structure and function within PAH and control groups and in the entire cohort (table 5 and figure 3). S2 complexity was increased in the PAH group in patients with moderate-to-severe RV enlargement and systolic 
A

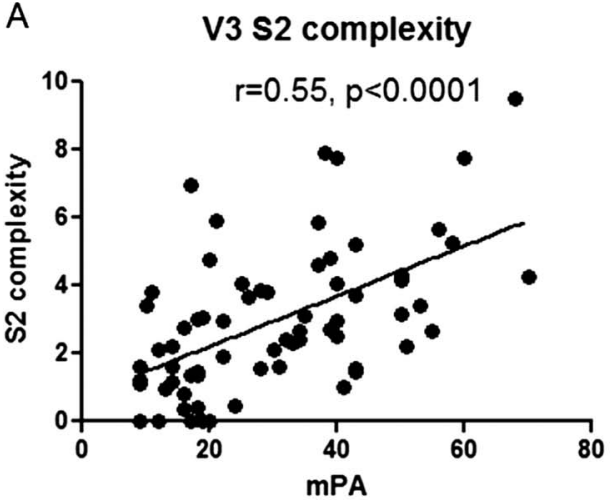

C

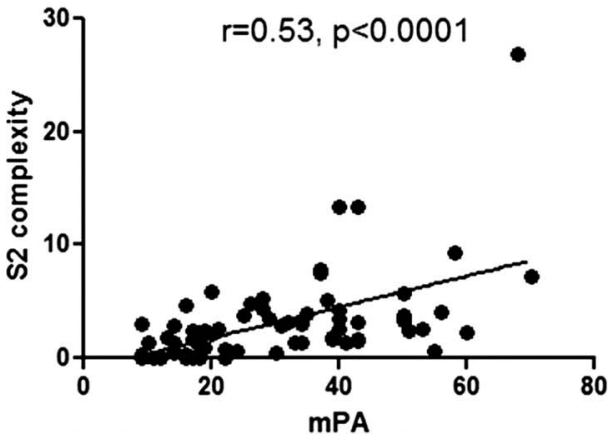

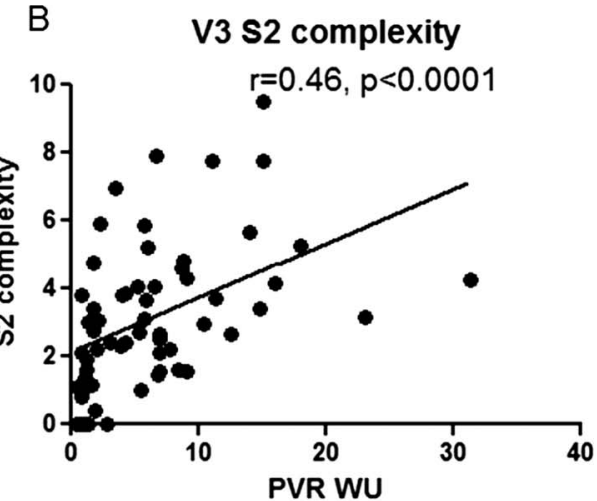

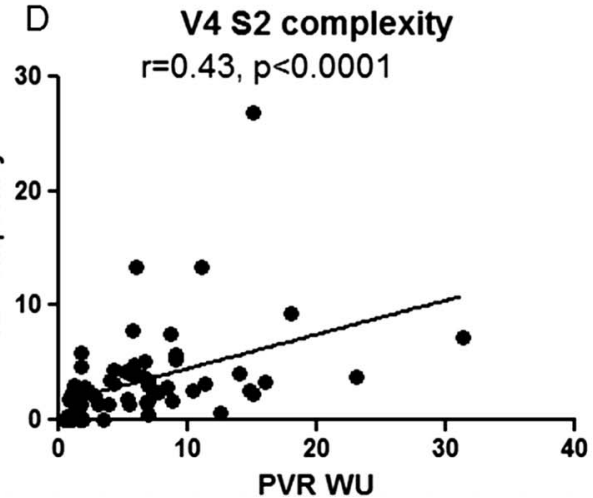

Figure 2 Relationship between S2 complexity and pulmonary vascular haemodynamics. mPA, mean pulmonary artery pressure; PVR, pulmonary vascular resistance; WU, Wood Units.

dysfunction compared with those with only normal or mild abnormalities. In particular, V3 S2 complexity was increased significantly in the PAH group among those with moderate-severe RV systolic dysfunction. In both V3 and V4 positions, in the entire cohort, S2 complexity remained clearly increased among those with moderatesevere RV enlargement and moderate-severe RV systolic dysfunction.

In contrast, S1 complexity showed an inverse relationship with increasing severity of RV enlargement and RV systolic dysfunction (table 5 and figure 3). In patients with $\mathrm{PAH}$ and moderate to severe RV enlargement and systolic dysfunction, V4 S1 complexity was reduced significantly compared with those with normal or mild RV enlargement and systolic dysfunction. This observation remained significant when the entire cohort was analysed. No such relationship between RV structure and function with S1 and S2 complexity was noted in controls.

\section{Evidence of ventricular interaction in PAH by acoustic cardiography}

We observed a direct relationship between increased $\mathrm{mPA} / \mathrm{PVR}$ and S2 complexity (figure 2). S1 complexity was inversely correlated with increasing severity of RV enlargement and RV systolic dysfunction (table 5 and figure 3). We hypothesised that these observations and the value of the S2/S1 complexity ratio may represent an acoustic corollary of the ventricular interaction in the
PAH group. Indeed, there was a direct relationship between S1 complexity in both precordial leads and LV end-diastolic diameter (figure 4A). We also observed a significant inverse correlation between V3 S1 complexity and V4 S2 complexity in the PAH cohort, $\mathrm{R}=-0.32$, $\mathrm{p}=0.049$ (figure $4 \mathrm{~B}$ ).

Value of quantitative acoustic variables for the detection of $\mathrm{PAH}$

We investigated the value of S2 complexity and S2/S1 complexity ratio for the detection of PAH $(\mathrm{mPA} \geq 25 \mathrm{~mm} \mathrm{Hg}$ ) by constructing ROC curves to assess the predictive performance in the $40 \mathrm{PAH}$ cases and 28 controls with haemodynamic data. As shown in figure 5, the AUC ROC curve for V3 and V4 S2 complexity was 0.80 and 0.85 , respectively. The optimal S2 complexity cut-offs for the discrimination of PAH was 1.44 (sensitivity $81 \%$, specificity $70 \%$, likelihood ratio 2.70 ) and 2.06 (sensitivity $70 \%$, specificity $87 \%$, likelihood ratio 5.27 ) for V3 and V4 S2 complexity, respectively. When we examined the S2/S1 complexity ratio, the AUC ROC was 0.89 ; however, this was not statistically superior to the AUC ROC for V4 S2 complexity ( $\mathrm{p}=0.53)$.

\section{DISCUSSION}

In this prospective cohort study, we investigated the quantitative relationship between acoustic characteristics of S2 and S1 and the haemodynamic severity of PAH as 
Table 4 Univariate haemodynamic predictors of S1 and S2 complexity

\begin{tabular}{|c|c|c|c|}
\hline & B (unstd) & Cl & p Value \\
\hline \multicolumn{4}{|l|}{ V3 S2 complexity } \\
\hline Mean pulmonary artery pressure & 0.07 & 0.05 to 0.10 & $<0.0001$ \\
\hline Heart rate & -0.01 & -0.06 to 0.05 & 0.85 \\
\hline Pulmonary capillary wedge pressure & -0.11 & -0.25 to 0.03 & 0.13 \\
\hline Cardiac output (Fick) & -0.27 & -0.71 to 0.18 & 0.23 \\
\hline \multicolumn{4}{|l|}{ Right atrial pressure } \\
\hline Mean systemic artery pressure & 0.02 & -0.04 to 0.07 & 0.58 \\
\hline \multicolumn{4}{|l|}{ V4 S2 complexity } \\
\hline Mean pulmonary artery pressure & 0.14 & 0.08 to 0.19 & $<0.0001$ \\
\hline Heart rate & 0.03 & -0.09 to 0.15 & 0.63 \\
\hline Pulmonary capillary wedge pressure & -0.09 & -0.36 to 0.19 & 0.52 \\
\hline Cardiac output (Fick) & -0.46 & -1.53 to 0.62 & 0.40 \\
\hline Right atrial pressure & 0.04 & -0.20 to 0.28 & 0.74 \\
\hline Mean systemic artery pressure & 0.02 & -0.12 to 0.15 & 0.81 \\
\hline \multicolumn{4}{|l|}{ V3 S1 complexity } \\
\hline Mean pulmonary artery pressure & -0.01 & -0.04 to 0.02 & 0.46 \\
\hline Heart rate & -0.01 & -0.06 to 0.03 & 0.51 \\
\hline Pulmonary capillary wedge pressure & -0.09 & -0.21 to 0.03 & 0.15 \\
\hline Cardiac output (Fick) & 0.37 & $0.02-0.72$ & 0.04 \\
\hline Right atrial pressure & -0.13 & -0.24 to -0.02 & 0.02 \\
\hline Mean systemic artery pressure & 0.04 & -0.01 to 0.08 & 0.11 \\
\hline \multicolumn{4}{|l|}{ V4 S1 complexity } \\
\hline Mean pulmonary artery pressure & -0.04 & -0.07 to -0.004 & 0.03 \\
\hline Heart rate & -0.03 & -0.08 to 0.02 & 0.24 \\
\hline Pulmonary capillary wedge pressure & 0.09 & -0.06 to 0.23 & 0.23 \\
\hline Cardiac output (Fick) & 0.49 & 0.04 to 0.93 & 0.03 \\
\hline Right atrial pressure & -0.10 & -0.22 to 0.03 & 0.12 \\
\hline Mean systemic artery pressure & 0.02 & -0.04 to 0.07 & 0.57 \\
\hline
\end{tabular}

well as the presence of RV dilation and systolic dysfunction. We demonstrated that the normal acoustic profile observed in controls, of increased S1 intensity/complexity compared to that of S2, was altered in the PAH group. S2 complexity was increased in the PAH group, and was directly related to $\mathrm{mPA}$ and PVR. Importantly, among PAH patients, the reciprocal relationship between S1 and S2 complexity likely represents the acoustic corollary of ventricular interaction that develops as the RV remodels during the course of PAH. These findings extend our mechanistic understanding of the relationship between pulmonary vascular and ventricular haemodynamics and the precordial acoustic profile.

Landmark investigations from the 1960s examined analogue phonocardiographic recordings and emphasised the relative amplitude of the aortic and pulmonic component of S2 (A2 and P2). ${ }^{5}$ The pulmonic component was said to be accentuated, reflecting the forceful closure of the pulmonary valve with the associated leftparasternal heave indicating RV pressure overload. An audible right-sided S3 and S4 was thought to indicate RV volume and pressure overload, respectively. ${ }^{10}$ Sutton et at ${ }^{\ominus}$ studied 116 patients. Twelve were classified as primary $\mathrm{PH}$ by cardiac catheterisation though only $50 \%$ of this cohort exhibited a P2 of higher amplitude than A2 by phonocardiography. Studies from this era acknowledged the challenges of discriminating A2 and
P2 by auscultation, and qualitative assessment of the summated S2 was also advocated. Thus, the detection of P2 in the mitral area ${ }^{9}$ or the relative loudness of S2 in the left versus the right parasternal second intercostal spaces $^{5}$ was also shown to be common in PH. These studies described the auscultatory or phonocardiographic findings prior to the systematic classification of PAH. ${ }^{5} 911$ Thus, classic lessons describing clinical auscultation findings of $\mathrm{PH}$ might have been confounded by the presence of the associated cardiac abnormalities such as left-to-right shunting or LV systolic dysfunction.

In the National Institutes of Health-sponsored Primary Pulmonary Hypertension Registry, abnormal auscultatory findings were highly prevalent. An increase in $\mathrm{P} 2$ of S2 was reported in 93\% of $187 \mathrm{PAH}$ patients. ${ }^{10}$ Cardiac auscultation is a highly cognitive process influenced by patient factors as well as the heuristic bias of the practitioner, contributing to poor interobserver agreement. ${ }^{12}$ In our study, the wavelet-based signal processing analysis of continuous digital acoustic data yielded a measurement not only of amplitude or intensity but also of time, energy and frequency characteristics, ${ }^{7}$ termed complexity. Previous studies have validated the detection of the S3 and S4 sounds by this methodology against echocardiographic- ${ }^{13}$ and catheterisation-based haemodynamic evidence of LV systolic dysfunction and elevated filing pressures. ${ }^{14-18}$ 
Table 5 S2 and S1 complexity according to right ventricular size and systolic function

\begin{tabular}{|c|c|c|c|c|}
\hline & \multicolumn{2}{|l|}{ RV dilation } & \multicolumn{2}{|c|}{ RV systolic dysfunction } \\
\hline & Normal/mild & Moderate/severe & Normal/mild & Moderate/severe \\
\hline \multicolumn{5}{|l|}{ S2 complexity } \\
\hline All (PAH and Controls) (n) & 37 & 23 & 43 & 17 \\
\hline V3 & $2.71 \pm 1.99$ & $3.89 \pm 2.14^{*}$ & $2.55 \pm 1.68$ & $4.85 \pm 2.20^{\star \star *}$ \\
\hline V4 & $2.63 \pm 2.40$ & $4.89 \pm 5.88^{\star}$ & $2.49 \pm 2.57$ & $5.74 \pm 6.33^{\star *}$ \\
\hline $\mathrm{PAH}(\mathrm{n})$ & 20 & 20 & 23 & 16 \\
\hline V3 & $3.33 \pm 1.66$ & $4.12 \pm 2.18$ & $2.95 \pm 1.21$ & $4.97 \pm 2.21^{\star *}$ \\
\hline V4 & $3.64 \pm 2.54$ & $5.53 \pm 6.05$ & $3.50 \pm 2.89$ & $6.10 \pm 6.36$ \\
\hline Controls (n) & 17 & 3 & 20 & 1 \\
\hline V3 & $1.98 \pm 2.15$ & $2.39 \pm 1.02$ & $2.08 \pm 2.04$ & $2.96 \pm 0.00$ \\
\hline V4 & $1.45 \pm 1.57$ & $0.59 \pm 1.02$ & $1.32 \pm 1.52$ & $0.00 \pm 0.00$ \\
\hline \multicolumn{5}{|l|}{ S1 complexity } \\
\hline All (PAH and Controls), (n) & 37 & 23 & 42 & 17 \\
\hline V3 & $4.55 \pm 2.06$ & $3.97 \pm 1.32$ & $4.60 \pm 2.00$ & $3.67 \pm 1.14$ \\
\hline V4 & $4.95 \pm 2.23$ & $3.81 \pm 1.34^{*}$ & $4.93 \pm 2.13$ & $3.69 \pm 1.35^{\star}$ \\
\hline $\mathrm{PAH},(\mathrm{n})$ & 20 & 20 & 23 & 16 \\
\hline V3 & $4.78 \pm 1.78$ & $4.11 \pm 1.30$ & $4.97 \pm 1.77$ & $3.80 \pm 1.05^{\star}$ \\
\hline V4 & $5.48 \pm 2.32$ & $3.68 \pm 1.21^{\star \star}$ & $5.29 \pm 2.20$ & $3.68 \pm 1.40^{*}$ \\
\hline Controls, (n) & 17 & 3 & 20 & 1 \\
\hline V3 & $4.29 \pm 2.38$ & $3.08 \pm 1.24$ & $4.19 \pm 2.20$ & $1.68 \pm 0.00$ \\
\hline V4 & $4.33 \pm 2.02$ & $4.68 \pm 2.08$ & $4.52 \pm 2.04$ & $3.83 \pm 0.00$ \\
\hline \multicolumn{5}{|c|}{$\begin{array}{l}{ }^{*} \mathrm{p}<0.05 . \\
{ }^{* \star} \mathrm{p}<0.01 \text {. } \\
\text { Comparison of heart sound complexity is performed between } \mathrm{PAH} \text { and controls as a cohort, and within the PAH and control groups based on } \\
\text { RV size and systolic dysfunction. } \\
\text { PAH, pulmonary arterial hypertension; RV, right ventricular. }\end{array}$} \\
\hline
\end{tabular}

In the current study, we examined the haemodynamic and structural correlates of digitally recorded precordial acoustic characteristics in PAH. S2 intensity was actually similar between the PAH and control groups. It is well understood that the intensity of precordial heart sounds is influenced by anatomic factors such as BSA, characteristics of the thoracic cage and cardiac axis. ${ }^{5}$ Beyond intensity, we observed that the complexity of S2 was significantly increased in $\mathrm{PAH}$ patients, consistent with the observation that $\mathrm{S} 2$ is frequently perceived to be abnormal in this condition. ${ }^{9}$ We also demonstrated that the perceived audible characteristics of $\mathrm{S} 2$ may in fact relate to the acoustic characteristics of $\mathrm{S} 1$. The ratio of $\mathrm{S} 2 / \mathrm{S} 1$ complexity was shown to be strikingly increased in the $\mathrm{PAH}$ group, interestingly with the leads in the precordial V3 and V4 position. This observation may be consistent with the older concept that P2 is frequently detectable in the mitral area in PH. Moreover, we observed a reciprocal relationship between $\mathrm{S} 2$ and $\mathrm{S} 1$ complexity that may have been related to ventricular interaction in $\mathrm{PAH}$ patients. Thus, the perception of an abnormally accentuated S2 may also relate to the abnormally diminished complexity or the audibility of $\mathrm{S} 1$.

We observed that mPA was an independent multivariate haemodynamic predictor of S2 complexity, consistent with other studies examining S2 acoustic qualities and TTE or RHC-derived pulmonary artery pressure. ${ }^{19} 20$ These findings provide support for the traditional construct that elevated PVR causes forceful closure of the pulmonic valve. We made several observations regarding the complexity of $\mathrm{S} 1$. In contrast to $\mathrm{S} 2$ complexity, mPA pressure was correlated with decreased S1 complexity. Worsening RV enlargement and systolic dysfunction were related to not only increased S2 complexity but also decreased S1 complexity. Diminished S1 complexity appeared related to decreased LV filling as measured by left ventricular end-diastolic diameter which may account for the observation that cardiac output was also independently associated with S1 complexity. LV underfilling may decrease the complexity of $\mathrm{S} 1$ as a result of either decreased LV force generation ${ }^{21} 22$ and/or the narrower separation of the mitral leaflets at the time of valve closure. $^{2324}$ The PR interval is a known determinant of S1 loudness, ${ }^{23} 24$ but was not statistically different between $\mathrm{PAH}$ and control groups. These observations suggest that the S2/S1 complexity ratio likely represents both the effect of PVR on pulmonic valve closure (increased S2 complexity) and reciprocal RV enlargement-LV underfilling (decreased S1 complexity). ${ }^{21} 22$ This concept of reciprocal ventricular interaction is well described in patients with $\mathrm{PH},{ }^{25-27}$ for whom we have now described the precordial acoustic corollary.

\section{LIMITATIONS}

Several limitations merit consideration. The sample size of the control cohort with both haemodynamics and echocardiography was relatively small, and we did not 
A
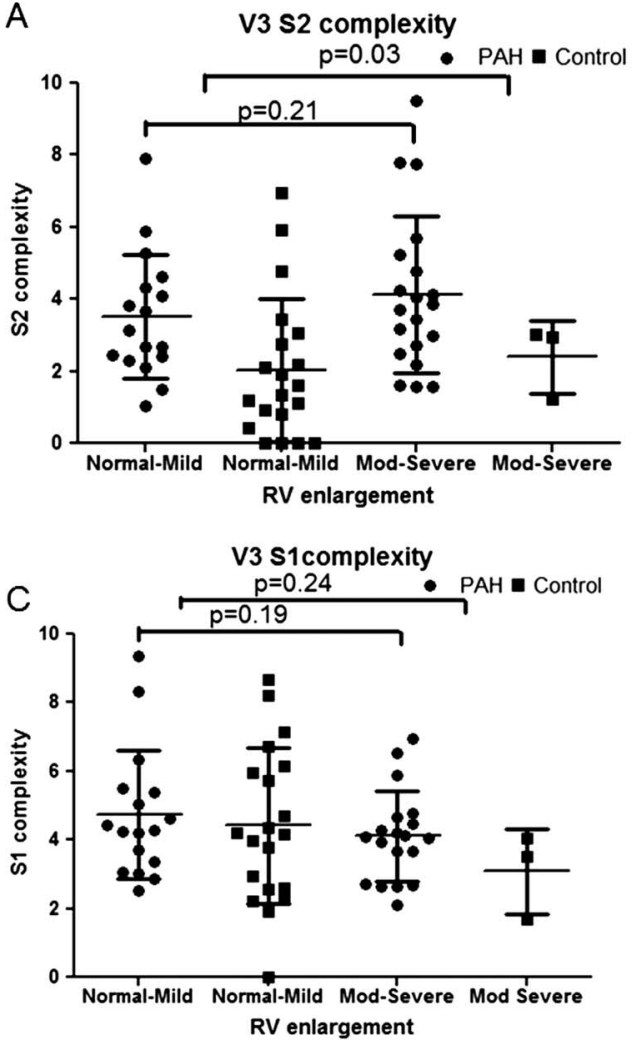

B
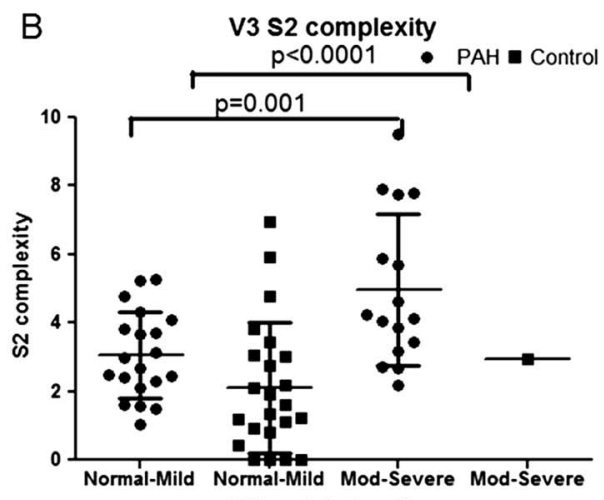

RV systolic function

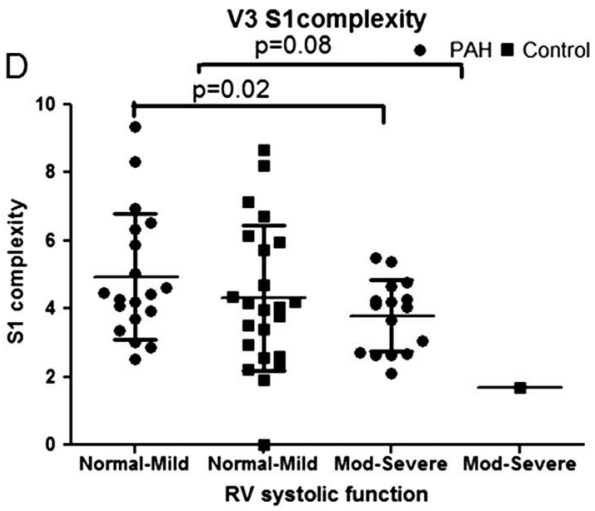

Figure 3 V3 S2 and S1 complexity according to right ventricular size and systolic function. V3 S2 complexity stratified according to severity of RV enlargement (A) and RV systolic dysfunction (B). V3 S1 complexity stratified according to severity of RV enlargement (C) and RV systolic dysfunction (D). Comparison of heart sound complexity is performed within the PAH group and also between PAH and controls as a cohort based on RV size and systolic dysfunction. PAH, pulmonary arterial hypertension; $\mathrm{RV}$, right ventricular.

match the PAH group against age and sex match. However, we adjusted for possible intersubject anthropomorphic differences by calculating the S2/S1 intensity and complexity ratios. Whether haemodynamic intervention and alteration of ventricular interaction influenced acoustic variables was not examined. Although S2 complexity and S2/S1 complexity ratio displayed potential predictive utility for the discrimination of $\mathrm{PAH}$, the study cohort was preselected to elucidate the physiological underpinnings of transmitted heart sounds, and thus patients with conditions that could be confused with PAH were excluded. Finally, the implication of our findings for clinical practice is unclear at present because the assessment and management of patients with $\mathrm{PAH}$ is guided more by functional class and RV remodelling.

\section{CONCLUSION}

The acoustic characteristics of S2 and S1 correlated significantly with the severity of PAH and evidence of RV dilation and systolic dysfunction. The reciprocal S2 and S1 complexity relationship among patients with $\mathrm{PAH}$ likely reflects ventricular interaction due to progressive RV remodelling. Our findings demonstrate that the perception of an abnormally accentuated S2 in PAH may be
Figure 4 Relationship between S1 complexity and left ventricular filling and S2 complexity. Significant correlation between V4 S1 complexity and left ventricular end-diastolic diameter (LVEDD) as a measure of left ventricular filling (A). Inverse correlation between S1 and S2 complexity measures suggests the presence of ventricular interaction in the $\mathrm{PAH}$ cohort (B).
A

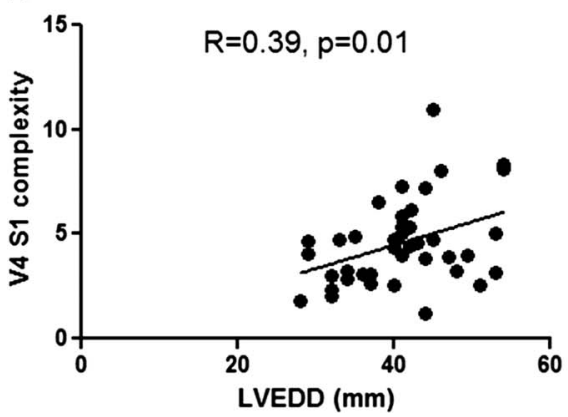

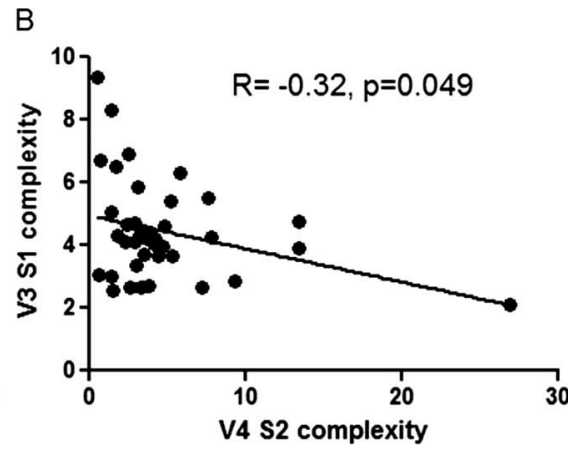


A

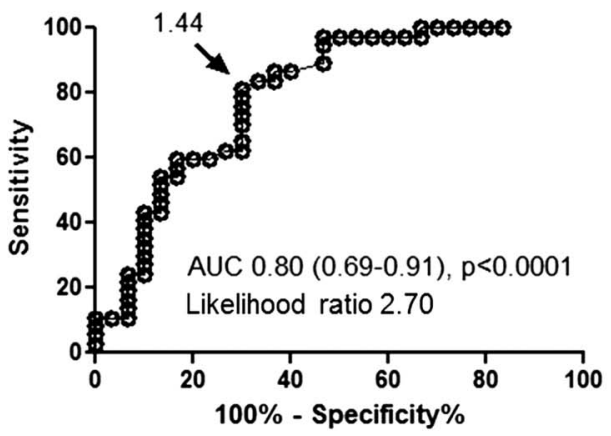

C

V3 S2/S1 complexity

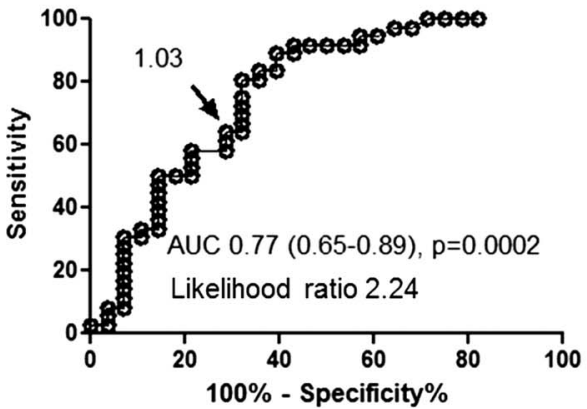

B

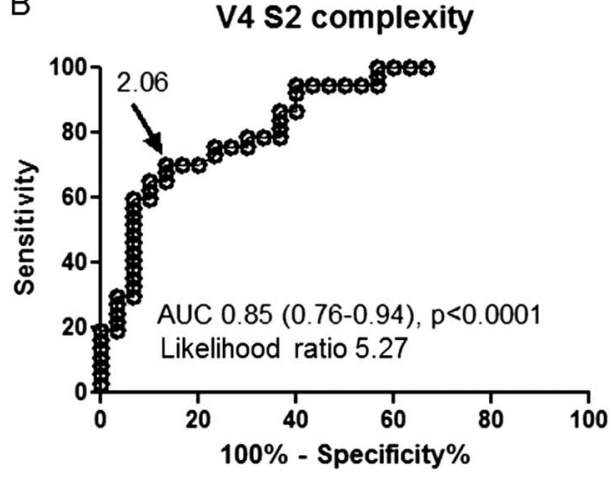

D

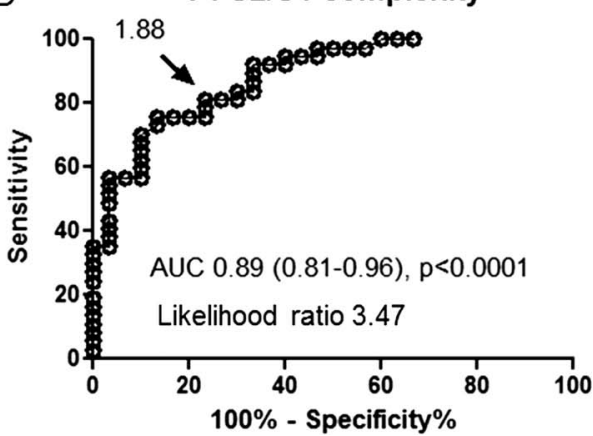

Figure 5 S2 and S2/S1 complexity receiver-operating characteristic curves for the detection of pulmonary arterial hypertension (mean pulmonary artery pressure $\geq 25 \mathrm{~mm} \mathrm{Hg}$ ). Receiver-operating characteristic curves for V3 S2 complexity (A); V4 S2 complexity (B); V3 S2/S1 complexity ratio (C) and V4 S2/S1 complexity ratio (D) for the detection of pulmonary arterial hypertension. AUC, area-under-curve.

derived both from not only increased S2 complexity but also diminished complexity or audibility of S1.

\section{Author affiliations}

${ }^{1}$ Division of Cardiology, Mount Sinai Hospital, Toronto, Ontario, Canada

${ }^{2}$ University of Toronto, Toronto, Ontario, Canada

${ }^{3}$ Inovise Medical, Inc., Beaverton, Oregon, USA

${ }^{4}$ Division of Cardiology, St. Joseph Hospital, Eureka, California, USA

${ }^{5}$ Division of Respirology, Toronto General Hospital, University Health Network, Toronto, Ontario, Canada

Contributors WC and SM take responsibility for (are the guarantors of) the content of the manuscript, including study design, manuscript preparation and revision and data collection and analysis. MW, RC, PA, ADM, JDP and JTG contributed to data analysis, manuscript preparation and critical revision. All authors have read and approved the manuscript for publication.

Funding This research received no specific grant from any funding agency in the public, commercial or not-for-profit sectors.

Competing interests PA is an employee of Inovise Medical. However, Inovise Medical Inc was not involved in the design of the study, data management, data analysis or manuscript preparation. Inovise Medical Inc was allowed to review the manuscript but any decisions regarding manuscript revision were made by the authors. WC is supported by the National Health and Medical Research Council of Australia Early Career Fellowship (Neil Hamilton FairleyClinical Overseas Fellowship) (APP1052960). SM is supported by a Heart and Stroke Foundation of Ontario (grant \# NA5579).

Provenance and peer review Not commissioned; externally peer reviewed.

Data sharing statement No additional data are available.

\section{REFERENCES}

1. Runo JR, Loyd JE. Primary pulmonary hypertension. Lancet 2003;361:1533-44.
2. McLaughlin VV, McGoon MD. Pulmonary arterial hypertension. Circulation 2006;114:1417-31.

3. Simonneau G, Robbins IM, Beghetti M, et al. Updated clinical classification of pulmonary hypertension. J Am Coll Cardiol 2009;54 (1 Suppl):S43-54.

4. Benza RL, Miller DP, Gomberg-Maitland M, et al. Predicting survival in pulmonary arterial hypertension: insights from the registry to evaluate early and long-term pulmonary arterial hypertension disease management (REVEAL). Circulation 2010;122:164-72.

5. Perloff JK. Auscultatory and phonocardiographic manifestations of pulmonary hypertension. Prog Cardiovasc Dis 1967;9:303-40.

6. McGoon M, Gutterman D, Steen V, et al. Screening, early detection, and diagnosis of pulmonary arterial hypertension: ACCP evidence-based clinical practice guidelines. Chest 2004;126(1 Suppl):14S-34S.

7. Erne P. Beyond auscultation-acoustic cardiography in the diagnosis and assessment of cardiac disease. Swiss Med Wkly 2008;138:439-52.

8. Lang RM, Bierig M, Devereux RB, et al. Recommendations for chamber quantification: a report from the American Society of Echocardiography's Guidelines and Standards Committee and the Chamber Quantification Writing Group, developed in conjunction with the European Association of Echocardiography, a branch of the European Society of Cardiology. J Am Soc Echocardiogr 2005;18:1440-63.

9. Sutton G, Harris A, Leatham A. Second heart sound in pulmonary hypertension. Br Heart $J$ 1968;30:743-56.

10. Rich S, Dantzker DR, Ayres SM, et al. Primary pulmonary hypertension. A national prospective study. Ann Intern Med 1987;107:216-23.

11. Wood P. Pulmonary hypertension. Br Med Bull 1952;8:348-53.

12. Marcus GM, Vessey J, Jordan MV, et al. Relationship between accurate auscultation of a clinically useful third heart sound and level of experience. Arch Intern Med 2006;166:617-22.

13. Zuber M, Kipfer P, Attenhofer Jost C. Systolic dysfunction: correlation of acoustic cardiography with Doppler echocardiography. Congest Heart Fail 2006;12(Suppl 1):14-18.

14. Collins SP, Arand P, Lindsell CJ, et al. Prevalence of the third and fourth heart sound in asymptomatic adults. Congest Heart Fail 2005;11:242-7.

15. Peacock WF, Harrison A, Maisel AS. The utility of heart sounds and systolic intervals across the care continuum. Congest Heart Fail 2006;12(Suppl 1):2-7. 
16. Shapiro M, Moyers B, Marcus GM, et al. Diagnostic characteristics of combining phonocardiographic third heart sound and systolic time intervals for the prediction of left ventricular dysfunction. J Card Fail 2007:13:18-24.

17. Efstratiadis $\mathrm{S}$, Michaels $\mathrm{AD}$. Computerized acoustic cardiographic electromechanical activation time correlates with invasive and echocardiographic parameters of left ventricular contractility. J Card Fail 2008;14:577-82.

18. Marcus GM, Gerber IL, McKeown BH, et al. Association between phonocardiographic third and fourth heart sounds and objective measures of left ventricular function. JAMA 2005;293:2238-44.

19. Chen D, Pibarot P, Honos G, et al. Estimation of pulmonary artery pressure by spectral analysis of the second heart sound. $A m \mathrm{~J}$ Cardiol 1996;78:785-9.

20. Michaels AD, Karabsheh S, Neuharth RM, et al. Non-invasive computerized acoustic cardiographic prediction of pulmonary hypertension. J Electrocardiogr 2009;42:617-18.

21. Sakamoto T, Kusukawa R, Maccanon DM, et al. Hemodynamic determinants of the amplitude of the first heart sound. Circ Res 1965;16:45-57.
22. Genest J Jr., Durand LG. Relationship of the left ventricular and apical first sounds to the left ventricular pressure derivative. Med Biol Eng Comput 1985;23:95-8.

23. Leech G, Brooks N, Green-Wilkinson A, et al. Mechanism of influence of PR interval on loudness of first heart sound. Br Heart $J$ 1980;43:138-42.

24. Stept ME, Heid CE, Shaver JA, et al. Effect of altering P-R interval on the amplitude of the first heart sound in the anesthetized dog. Circ Res 1969;25:255-63.

25. Dong SJ, Crawley AP, MacGregor JH, et al. Regional left ventricular systolic function in relation to the cavity geometry in patients with chronic right ventricular pressure overload. A three-dimensional tagged magnetic resonance imaging study. Circulation 1995;91:2359-70.

26. Marcus JT, Vonk Noordegraaf A, Roeleveld RJ, et al. Impaired left ventricular filling due to right ventricular pressure overload in primary pulmonary hypertension: noninvasive monitoring using MRI. Chest 2001;119:1761-5.

27. Gurudevan SV, Malouf PJ, Auger WR, et al. Abnormal left ventricular diastolic filling in chronic thromboembolic pulmonary hypertension: true diastolic dysfunction or left ventricular underfilling? J Am Coll Cardiol 2007;49:1334-9. 